both mild and severe outbreaks, some of which will come under consideration in connection with milk and water epidemics. The death-rate from enteric fever has for many years been falling, and hospital statistics show that there has been some decline in severity of hospital cases, insufficient, however, to account for the less mortality. Apparently therefore there has been lessened prevalence as well. The statistics published by the Metropolitan Asylums Board show that the case mortality has varied considerably in different years, but neither the hospital records nor the death-rates show any clear trace of the five-yearly cycle which at one time was so conspicuous in regard to erysipelas, diphtheria, and scarlet fever. On the whole, therefore, enteric fever may be regarded as decreasing in severity and still more in prevalence, but exhibiting irregular fluctuations in both respects.

Many other examples might be given, but the above may serve to illustrate the broad principle that the contagia which are most changeable in type are those which remain in closest touch with the saprophytic forms to which they are allied.

\section{ON THE TREATMENT OF COMPLETE OBSTRUC-}

\section{TION OF THE LARGE INTESTINE BY} TEMPORARY TYPHLOTOMY.

\author{
BY HARRISON CRIPPS, F.R.C.S., \\ Assistant Surgeon to st. Bartholomew's Hospital.
}

THr train of symptoms arising from complete obstruction of the large intestine is such that in the majority of cases a differential diagnosis can be clearly made between obstructions in this part of the bowel and the small intestine. It will be well to consider a few of the broader features which point to the obstruction being in the large rather than in the small intestine.

\section{History of thr AtTack.}

Speaking generally, all the symptoms are more sudden and acute in obstructions of the small than of the large bowel. Thus in the former the symptoms may be reckoned by hours and days, while in the latter it is rather by days and weeks. Hence the terms which some writers employ, acute intestinal obstruction and chronic intestinal obstruction, the former referring to the small, the latter to the large intestine. It is possible that the nervous mechanism of the upper bowel is more sensitive to injury, but it is probable that the difference in severity arises more from the cause of the obstruction than from its actual position. In far the larger number of instances the cause of obstruction to the small intestine arises external to the gut-twists, herniæ through the omentum and mesentery, or slipping of the gut beneath old bands of adhesions. In such accidents not merely is the calibre of the bowel obstructed, but its vessels are strangulated, and the experience of ordinary hernix demonstrates clearly that the severity of the symptoms is in proportion to the degree to which the bowel is nipped. Obstruction of the large intestine, on the other hand, most commonly results from obstruction within - strictures fibrous or malignant, impacted fæces, etc. Indeed, very commonly it is a combination of the two. Thus, a bowel may be considerably narrowed by stricture, and yet give rise to no severe symptoms till a scybalous mass accidentally becomes jammed into its upper orifice. Occasionally the large intestine, or more commonly its sigmoid flexure, may be the seat of volvulus, when the symptoms are often as acute as if the small intestine were implicated. The acute symptoms consequent on obstruction of the small intestine are generally ushered in with severe pain and violent vomiting, and the patient quickly passes into a state of collapse; unless the obstruction is low down there is comparatively little abdominal distension. When the obstruction is in the large bowel the onset is more insidious. Vague colicky pains, with obstinate constipation and a feeling of nausea, may be all that is complained of the first few days. Pargative medicine may have been taken, coincidently with which the severity of the symptoms increase; vomiting is slight at first, merely consisting of the contents of the stomach and duodenum, seldom becoming fæcal till at

\footnotetext{
1 Read before the Harveian societs.
}

least four or five days after its onset. Day by day the abdomen becomes more and more distended and tympanitic, and if unrelieved the patient gradually passes into a state of collapse. Although in most cases of obstruction of the large intestine the symptoms are comparatively chronic, there is great variation in the interval which elapses between the first onset of the symptoms and their fatal termination. Sometimes a patient may be in an extreme condition after a week or ten days, while in a few instances a month or even longer may elapse before the general symptoms become urgent. In such cases, however, the obstruction is generally quite low down-in the rectum.

Intussusception, with its characteristic symptoms, belongs both to the small and large intestine; its treatment, therefore, will not be considered in this paper. Nor dc I propose to go further into the details of the symptoms of obstruction.

It is assumed that we are now at the bedside of a patient who is obviously suffering from obstruction, and the general symptoms point to the large intestine as being its probable seat. The symptoms have been present for some days, and have been gradually increasing in severity. A careful examination of the abdomen has revealed no definite tumour or other indication suggesting the exact site of the obstruction, and an examination of the rectum by the finger has given merely negative results. I may here say that the evidence afforded by the passage of tubes up the bowel is absolutely worthless, and that their employment is not devoid of danger. I have been present when some feet of soft rubber tubing or œsophageal bougie have been triumphantly passed up the rectum, and the procedure regarded as evidence that the obstruction must be at least as high as the transverse colon. Nevertheless, post-mortem examination showed an impassable stricture in the upper part of the rectum. Fortunately, these tubes bend and coil up, otherwise, if any force were used, they would certainly perforate the bowel. Even when no obstruction exists, I suspect that few tubes have ever been passed as high as the sigmoid flexure, and probably never beyond it.

Treatment.

Injections.-Since many cuses of obstruction are due to a scybalous mass impacted into a previously narrowed or strictured portion of the bowel, the effect of carefully administered injections should be tried with a view to the washing away or breaking up of the obstructing plug. Injections should be used on a regular plan. I pass into the rectum, as far as it will go without using the slightest force, a half.inch india-rubber tube. It is then cut off so that only a few inches protrude from the anus. The patient being placed on his side at the edge of the bed, as much warm water as the bowel will hold without undue pressure is injected slowly up the tube by means of a Higginson's syringe. This having remained a few minutes, the nozzle of the syringe is removed, and the water allowed to return through the tube. This water is usually mixed with a certain amount of fæcal debris which has collected below the obstruction. A second quantity of water is now injected. If this returns clear, it is probable that little good will result from the washing, although it may be repeated once or twice again. On the other hand, should it return markedly stained the case is more hopeful, for it may mean that the water is disintegrating the lower part of a fæcal block. Indeed, so long as the water returns stained the injections should be repeated, and in fortunate cases it may happen that after a few washings the obstruction gives way, and copious evacuations follow. Warm water seems preferable to oil as having more solvent properties, and it also enables the effect of the injections to be more accurately gauged by observing the extent to which it is stained. We will now suppose that the injections have afforded no relief, and the question arises as to what further treatment can be adopted. Of course it is possible that, if untreated surgically, the obstruction may give way by the aid of Nature alone, but the question for decision is whether a better chance is given to the patient by leaving the case to Nature, or by direct surgical interference. Provided the patient be not in extremis, $I$ have no hesitation in strongly urging operative measures as affording the patient the better chance.

The Selaction of an Operation.

An operation being dẹçided upon, the best manner of pro- 
ceeding alone remains for consideration. If an obstruction be felt in the rectum or a tumour in the abdomen an indication will be afforded as to the locality for operation, but we will assume that we have a case, such as so commonly presents itself, in which, though the general symptoms point to obstruction in the large intestine, there is nothing to indicate more exactly its site. Pathological experience affords us some help, for it teaches that in three cases out of four the obstruction will be found on the left side in the neighbourhood of the sigmoid flexure. Some surgeons open the abdomen in the middle line as affording the best means of exploring all the large gut with a view to the possible excision of the obstructing part and the production of an anastomosis. The necessities of the case, however, often demand a departure from the theoretically ideal treatment. Many of the patients are in such a condition as would forbid any prolonged or hazardous operation. The excision of a portion of the bowel and the joining of the divided ends may be an ad. mirable operation if undertaken at the right time, but to attempt this on a patient suffering from the shock of fæcal vomiting and with a belly distended like a drum is almost certain to deprive him of his last chance of life. Even if the patient does not succumb from the shock of a prolonged operation and if the anastomosis is successfully accomplished, he is probably no better off than he was before. The intestine above the junction is dilated and paralysed and has no power of driving its contents through the contracted bowel below. The patient is consequently unrelieved. In all cases of complete obstruction of the large intestine it is of paramount importance to give immediate relief to the distended bowel. When this has been accomplished, as it certainly can be by a temporary colotomy or typhlotomy, the question of resecting the bowel can be deferred to a subsequent date, when it can be undertaken on an empty intestine with all due leisure and a prospect of success. Dismissing then for a time the intention of performing any elaborate operation. I would advocate that the abdomen should be opened in the first instance on the left side rather than in the middle line, for the following reasons :

1. Because in the majority of cases such an incision will bring the operator at once to the site of obstruction.

2 . If the obstruction is of a nature that necessitates colotomy the exploratory incision serves as a means of completing the operation.

3. If the obstruction is not found at the site expected the emptied sigmoid or descending colon at once shows that it is higher up and will almost certainly have to be dealt with by a temporary typhlotomy.

4. It must be remembered that in these cases the whole abdomen is tightly distended by inflated intestine and that it would often be impracticable to drag any part of the ascending or descending colon to an opening in the middle line without undue tension.

Ophration primarily Exploratory.

If it be decided to open on the left side, the best site for the incision is obtained by drawing an imaginary line from the anterior superior iliac spine to the umbilicus. The incision is bisected by this imaginary line at a distance of three fingers' breadth from the anterior spine. On opening the abdominal cavity the sigmoid flexure must be searched for, and will generally be found distended; and a little further examination will disclose the cause of obstruction, which must be dealt with according to its nature. Should it prove a volvulus it might be untwisted; if an impacted mass of fæces the bowel should be incised, and the impaction removed. Should, however, the obstruction, as most commonly happens, be caused by a strictured bowel, and the stricture of such a nature that no subsequent operation could be performed for its relief, a full-sized colotomy opening should be made to complete the treatment. If the obstruction prove to be a volvulus or impacted fæces, it would be a nice point to determine whether the untwisting of the volvulus or the removal of the impaction would be sufficient, or whether it might not be better, after relieving either of these two conditions, to stitch a small portion of the gut which had been distended above the obstruction to the skin, and make a small temporary opening into it in order to secure a means for the distended bowel to get rid of its gaseous and fæcal contents. It does not seem sufficient merely to tap the paralysed and distended bowel with a view to letting out its contents, and immediately closing the opening, for at first, as pointed out by Mr. Greig Smith, it is only a small segment of the gut which will discharge itself in this way, whereas if a small part of the bowel is stitched to the skin accurately, and opened, it will in the course of a few hours or days completely empty itself, and the small opening can be subsequently closed. The surgeon, in these circumstances, should be guided by the general condition of the bowel. If the obstruction be of long standing, it would probably be the wiser course to open the intestine. On the other hand, if the obstruction had only been for a short period, the bowel might be trusted to pass its contents forwards. This remark only applies to the left side, for I am confident that with obstructions higher up the bowel, where there is a longer piece of contracted and empty colon, it would be better to open it in every instance.

In a small portion of my cases, on exposing the colon on the left side it has been found empty and collapsed. Of course a moderate search should be made by introducing the fingers, and gently drawing down the bowel to ascertain whether the obstruction, though higher up, may yet be within reach. If this fails I would immediately close the abdominal incision with silkworm gut, and open the abdomen on the opposite side over the cæcum. In those cases in which I have done this the distended cæcum was found without difficulty, although on the first occasion it looked so like a distended stomach that I hesitated to open it. After an experience, however, of a few cases it can be confidently recognised. A portion of the cæcum, about the size of a sixpence, is carefully stitched to the parietal peritoneum with the finest silk sutures. If the abdominal walls be thin, the skin may be included in the sutures, but if the walls be fatty and thick it is unsafe to attempt to draw the bowel and peritoneum up to the skin, for the tension will probably cause the stitches to tear from the bowel. The parietal peritoneum can be accurately stitched round the bowel, so as effectually to shut off the general peritoneal cavity. The sutures, of course, are applied as in inguinal colotomy, and do not pass through the whole thickness of the gut. When the stitching is completed an opening that will admit the little finger is made into the exposed cæcum. If this be made in the first instance by a trocar and cannula passed through a piece of thin indiarubber sheeting the fluid freces can be conducted into a receptacle without flooding the wound and table. By the escape of air and fluid the distended cæcum and colon are quickly relieved, and, if peritonitis has not already occurred, the patient's life is for the time saved, and the subsequent treatment of the case can be considered at leisure. If the obstruction is of the nature of a volvulus, the distension behind being relieved, it has a fair chance of untwisting, for it has been shown by Treves and others how a volvulus is often prevented from untwisting by being pressed against the abdominal parietes by distended intestine. I have no doubt that this is the explanation of the extremely satisfactory result obtained in a case the short history of which I have appended to this paper. Again, should the obstruction have been caused by the impaction of a fæcal mass, time has been gained by opening the cæcum to allow of its gradual disintegration, and the restoration of a free passage through the bowel.

Lastly, should the obstruction have been due to some growth occluding the canal, the cæcal opening may be enlarged as a means of permanent relief, or, should the age of the patient admit, some subsequent operation might be undertaken with a view to excising the obstruction and establishing an anastomosis.

\section{SUMmarX}

I would advise that first in all cases of obstruation in the large intestine, when injections have failed, and where neither the exact site nor the cause can be diagnosed, that the abdomen should be opened on the left side over the sigmoid flexure, but if this part of the bowel prove to be below the obstruction the wound should be closed, and the cæcum exposed on the opposite side.

Secondly, I advise that a small opening should be made in the distended cæcum after stitching it to the parietal peritoneum; and that this opening may be nltimately enlarged or 
permanently closed according to the nature of the obstruction as shown by the subsequent progress of the case.

The two cases appended to this paper, both of which occurred in my practice in the same week, will serve as illustrations of the treatment advocated. In both, the symptoms were immediately relieved by the small opening into the crcum ; in both Nature ultimately restored the natural passage, and the fæcal fistulæ were closed by the simplest operation. As an illustration of how little shock is induced by the treatment suggested, I may mention that the second of my cases was a lady over 80 years of age, who completely recovered, and has since remained in excellent health.

CASE I.-In March, 1892, Dr. Jeaffreson, of Wandsworth, askad me to see a lady, aged 45, with the following history: On some four or five occasions during the last ten years she had had attacks of sharp colicty
pains, lasting a few hours and passing away without further symptoms. Seven days before she had had such an attack, but instead of the pain passing off as on former occasions it had remained, coming on in paroxysms. During the last two days she had had a certain amount of paroxysms. During the last two days she had had a certain amount of sickness commenced. She had several injections of water and oil, but practically without result. On my visit the patient expressed herself as preoling better, and in less pain than on thel previous day, and there had been no vomiting for the last twenty-four hours The temperature was normal, and the pulse 100. The abdomen was uniformly and greatly wollen, no tumour could be recognised. nor could anything be felt in the rectum. Owing to the improvement in the symptoms and vomiting havfood was stopped by the mouth, small nutrient enemata being food was stopped by the mouth, small nutrient enemata being substituted. During the next two days there was practically no change day, however, vomiting recommenced, and the belly appeared a little it was decided that operative interference should be no longer deforred.

The abdomen was opened by an incision three inches long on the loft side; distended small intestine immediately crowded out. Two fingers introduced could feel nothing but distended intestine. The empty as far as it could be examined. The wound was closed, buried sutures being used. The abdomen was then opened on tha opposite side over the cecum, and this was found enormously distended. The pach a way that only a small circle of gut, the size of a sixpence, was left exposed. The cæcum was then punctured by a trocar and cannula the puncture being made through a piece of india-rubher sheeting to protect the wound. A large quantity of wind and fluid frees escaped through the cannula. This at once caused the abdomen, which had been previously tightly distended, to collapse to a considerable extent, and opening so as to introduce the little fingar. Previous to puncturing the opening so as to hatroduce the litle fogar. Previous to puncturing the surface, but after its contents were evacuated it came to the skin level without any tension. Ten more sutures were then passed, fixing the small opening firmly to the skin

The general symptoms were at once relieved. and the patient made an uninterrupted convalescence. Fifteen davs after the operation a small quantity of motion was passed for the first time hy the rectum. The quantity then increased day by day. so that by the end of a month nearly the whole of the motion passed by the natural passage. The opening occasionally passed through this to the size or a vo. stity of fæcal discharge. In May I closed the fistula. The patient walked and drove about, and remained in good health till the end of July. She then had nnother attack exactly similar to the first. The abdomen became greatly distended. with much pain and vomiting. Simple measures having failed, and the patient on the fourth day passing obviously into a dangerous condition, I cut through the centre of the small scar, and again opened the crcum sufficiently to admit the little finger. A copious escape of wind and fluid fæces gave rapid relief. The patient convalesced in a few days, and normal motions again passed by the rectum. The patient now has a little valvular opening over the crecum, the size of a cally unstained. The patient will not have this closed at present, hut keeps it open by a little glass rod, passed daily. She says the opening causes her the least possible inconvenience, and she regards it as a safetyvalve against the recurrence of the symptoms which she so greatly dreads.

CASE Ir.-In March, 1892, I was asked by Dr. Townsend to see a patient, aged 79, in consultation. She had onjoyed good health till seven days previously. She then suffered a good deal of griping pain. Since that time she had passed no motion, and suffered severely from recurrent paroxysms of pain. During the last thirty-six hours she had had much sickness and retching. The abdomen was greatly distended and tympanitic. No tumour could be discovered either through the abdominal walls or by the rectum. We agreed that if by the following morning the symptoms were unrelieved an exploratory incision should be made. The symptoms having increased rather than otherwise, the abdomen was
opened on the left side in the position already described. Distended small intestine presented itself. On further search the lower part of the sigmoid flexure was found contracted and empty. On following this up for 8 or 9 inches, a hard solid mass was felt. With some little difficulty hard mass of inspissated fæces in the lower part of the descending colon. hard mass of inspissated frecs in the lower part of the descending colon. The mass was about 4 inches long and 2 in diameter. The colon was so stretched over it as to be semitransparent and scarcely thicker than incision 2 inches in length was made in the long dismeter of the bowel opposite the mesentery. The mass was then broken in two, and each portion extracted without difficulty. The bowel wound was closed with fifteen fine silk sutures, the temporary ligature removed, the intestine returned into the abdominal cavity and the wound

hours later I was summoned to see the patient again. Boon after the operation ghe had passed some wind and a small quantil. creased agin, and romiting continued I felt confident that the large intestine so long distended above the obstruction wis paralysed and un able to pass its contents through the contracted intestine lying below the obstruction, thus practically the patient was dying unrelieved. Her general condition was such as forbade further delay. or even an anæsthetic, so that at once, with the assistance of Dr. Townsend, I made a small incision into the abdominal carity, and exposed the cæcum. Which was greatly distended. The pariotal peritoneum was carefully stitched to this. so as to leave a circular area of the bowel no larger than a threepenny plece exposed. A trocar and cannula were then introduced into An india-rubber tube the size of the little finger was then passed into the opening, in which it fitted fairly tightly, and through this the bowel was washed out with a copious supply of hot water. By carefully packing sterilised wool round the tube, the wound itself was kept comparatively free from contact of facal material. The following morning all vomiting had ceased. and the patient's general condition had greatly improved. A large quantity of liquid fæces had passed through the opening. She made an uninterrupted recovery, motions beginning to be passed by the rectum on the eighth das. At the end of a month the small opening in the cæcum was closed by operation, and the patient, now over 80 years of age, when last see
excellent health.

\section{THE END OF A "PRACTICAL CURE."} [WrTh Chromo-Lithograph.]

By Sir SPENCER WELLS, BART., F.R.C.S., Surgeon to the Queen's Household.

IN the summer of 1879 I amputated the elongated and enlarged cervix uteri of a married lady who had suffered from supposed papilloma or epithelioma, and had been treated unsuccessfully by injection of perchloride of mercury into the substance of the cervix. Examination of the part of the cervix removed proved that the enlargement was due to adenoma, or simple hypertrophy of the utricular glands. The patient was much relieved by this amputation, but about a year afterwards the remains of the cervix showed some tendency to enlarge and proliferate, and I destroyed the affected part by the actual cautery. There was some pain and fever for a few days after this, but they soon subsided, and for about seven years afterwards, until 1887, she had as a rule excellent health, surprising her friends by her improved appearance; occasionally suffering from menorrhagia but at other times walking several miles without fatigue, driving, riding, rowing, dancing, and enjoying society more than most people. Her husband died in 1887, and in February, 1888, she married again. She remained very well during that summer, and in the autumn went to Scotland, where, after a fall or blow, she suffered considerable pelvic pain. She then travelled to Edinburgh and Wales, and afterwards to Birmingham in November. She was there assured that her life was in imminent danger, that immediate operation was necessary, that she would die or become insane if it were not done, that the risk was very small, the after pain insignificant, and a cure almost certain. Her ovaries and Fallopian tubes were removed. About a month after this operation the operator exhibited these ovaries and tubes before the British Gynæcological Society, and said the patient was then " practically cured." The uterine disease was entirely ignored, and I was accused of having overlooked a pyosalpinx due to a gonorrhœa from which she had suffered many years before. I took no notice of this attack for about a year, although I suspected a useless operation had been performed and the "cure" very doubtful.

Long before this I had repeatedly condemned a growing tendency to perform unnecessary mutilating operations upon women, both married and unmarried, and had protested against any teaching or example which might tend to lower the standard of surgical morality and to endanger the character of British surgery. Afterwards, when delivering the Bradshaw Lecture at the College of Surgeons, in December, 1890 , I expressed my fear that "the operation of oöphorectomy has been injudiciously performed without due explanation of its consequences, and with mistaken prophecies of 